\title{
Long-term stability of pairwise social dominance in squirrel monkeys*
}

\author{
DENNIS L. CLARK. KAREN L. KESSLER \\ and JOHN E. DILLON \\ Lniversity of Arizona. Tucson. Ariz. 85721
}

\begin{abstract}
Three separate measurements of pairwise social-dominance relations between 10 feral adult squirrel monkeys were conducted at 6 -month intervals. The monkeys were housed individually throughout the experiment and were undisturbed between the dominance tests. These conditions permitted the assessment of both short-term stability of individual animals' dominance behaviors and long-term stability of the group dominance structure in the absence of interpolated social experiences. Each dominance test consisted of observing all 45 possible pairs of monkeys in a competitive water-incentive situation. Dominance hierarchies were formed by ranking the animals on the basis of the total number of pairmates dominated within each separate test. A high level of stability in individual animals' dominance ability was observed within each of the three tests. Very high correlations were also obtained between successively measured dominance hierarchies. The status position changes which were observed in the successive dominance tests were very similar to changes reported for free-living squirrel monkey males observed under seminatural conditions.
\end{abstract}

Clark \& Dillon (1973a, b) recently studied social dominance relationships and incentive-getting behaviors shown by adult squirrel monkeys in a competitive water-incentive situation. Using 10 animals as Ss, these investigators observed highly stable dominance ability within individual monkeys measured in daily pairwise competition. For example, the most successful animal consistently defeated all 9 of his partners, the second ranking animal consistently defeated all partners except his immediate superior, and so on down the ranks of the dominance hierarchy. The effect of this consistency in dominance relations was to produce an essentially linear dominance hierarchy among the group of 10 animals, a result which confirmed the previous studies of Plotnik. King, \& Roberts (1968) and Candland, Bryan, Nazar. Kopf, \& Sendor (1970), who observed linear dominance in smaller squirrel monkey groups. The conclusion follows that adult squirrel monkeys exhibit stable linear dominance relationships, at least when measured in short-term or cross-sectional studies in the laboratory.

However, other studies involving longer time spans have indicated that squirrel monkey dominance relations may not be stable in a longitudinal sense. Ploog, Blitz, \& Ploog (1963) studied six squirrel monkeys living as a group for 12 months, and Baldwin (1968) spent 13 months observing a seminaturally living group of squirrel monkeys. Both of these investigators reported gradual changes in the frequency and direction of aggressive

*Sponsored by James E. King. who takes full editorial responsibility for its contents. behaviors among the monkeys in their groups. For example, Baldwin (1968) observed a total of seven different dominance orders among the four adult males in his group over the course of their 4-month breeding season, although at any given time the dominance hierarchy was linear in structure.

Since monkeys in the Ploog et al (1963) and the Baldwin (1968) studies lived under conditions of continuous social contact throughout the observation periods, it is possible that the dominance hierarchy changes resulted from specific winning and losing experiences among the animals involved. On the other hand, it is also possible that "spontaneous" changes, or changes caused by factors other than social experiences, might occur in a squirrel monkey dominance hierarchy over long periods of time during which the monkeys are segregated from each other. The present experiment was conducted to examine this latter possibility, that is, to assess the degree of spontaneous change within a squirrel monkey dominance hierarchy when the measurement occassions are separated by long periods of time involving no social contact between the monkeys.

\section{METHOD \\ Subjects}

Ths $\mathrm{Ss}$ in the present experiment were the 10 adult feral squirrel monkey's (Saimiri sciureus. Colombian variety). 5 males and 5 females, employed originally by Clark \& Dillon (1973a). These monkeys had lived in the laboratory for 5 months prior to the initial dominance measurement, and were housed individually in standard laboratory cages separated by Masonite panels. This home-cage condition was maintained throughout the 12 -month period of the present study. No social contact was allowed between the monkeys in the study, or with any other monkey, except during the dominance measurement sessions.

\section{Apparatus}

Dominance testing was carried out in a dominance apparatus which consisted of a standard $18 \times 27 \times 28$ in. laboratory cage devoid of perches. A water bottle was located on the rear wall of the cage in a hinged bottle holder that allowed the water spout to be moved in or out of the cage. at a distance of 8 in. from the cage floor, by the $E$. This test cage was situated in an experimental room separate from the main monkey colony.

\section{Procedure}

A more detailed description and evaluation of the dominance testing procedure appears in Clark \& Dillon (1973a). Briefly. the following procedure was employed in Dominance Tests I. II. and III in April. October. and April. respectively. During the 6-month periods between dominance tests. the animals were left undisturbed in their individual cages. except for routine cleaning and feeding.

Following home-cage pretraining to drink a blue-colored 15 ? (by volume) sucrose solution. a series of daily 5 -min nonsocial trials was given to each monkey in the dominance apparatus to habituate the animal to the drinking situation. These adaptation trials were continued until each $\mathrm{S}$ met the criterion of accumulating $15 \mathrm{sec}$ of drinking the blue-sweet incentive within the first $60 \mathrm{sec}$ of the 5 -min session on 3 of 4 consecutive dars 
Table 1

Results of Dominance Tests I. II. and III*

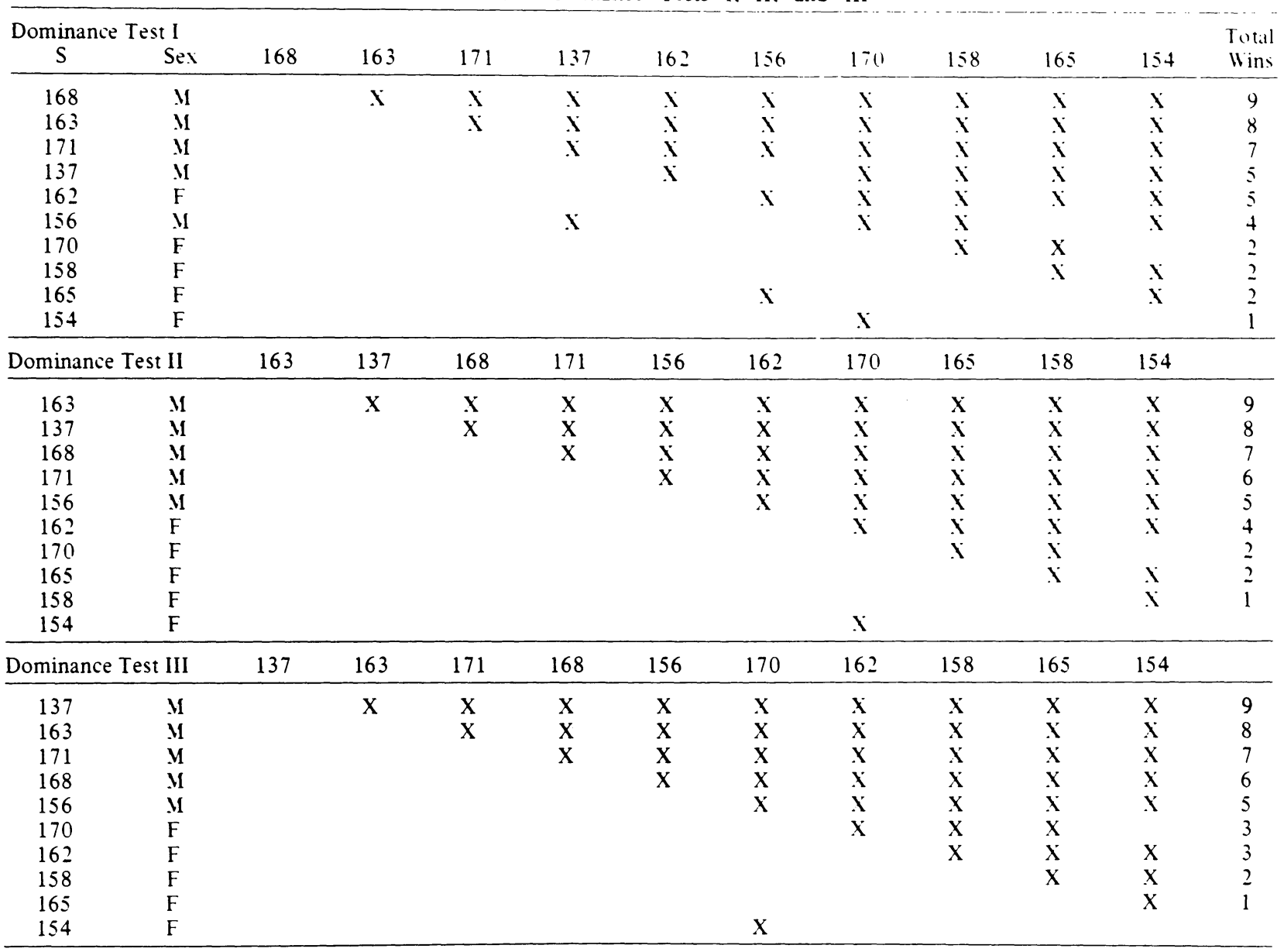

*An " $X$ " indicates that the monkey in the row dominated the monkey in the column.

All testing followed $6 \mathrm{~h}$ of water deprivation. A nonsocial trial consisted of bringing each monkey to the test chamber in a transport cage. placing him in the test cage. lowering the water spout into the test cage after a $30-\mathrm{sec}$ adjustment period. and recording the monkey's behavior during the subsequent 5-min trial. Trials were timed automatically. On the day prior to the start of social testing. all $10 \mathrm{Ss}$ were again tested nonsocially to promote equal habituation.

The social dominance trials were administered by testing the 10 monkeys in all possible pairwise combinations. This procedure resulted in a total of 45 pairings, given over 9 days of testing. for each of the three dominance tests. The testing and pairing schedule for each dominance test was determined randomly. with the restriction that a given monkey be tested only once per day. Each social trial was carried out in precisely the same manner as the nonsocial trials, except that the monkey's were observed in pairs. each animal scored by a separate $O$.

A win or loss was scored for both monkeys in each pairing immidiately following each trial. Dominance was awarded on the basis of a monkey's ability to maintain control over the water spout. or the area immediately surrounding the spout. by pushing. holding. chasing. or in some other manner actually displacing the submissive monkey from the water incentive. As previously reported (Clark \& Dillon. 1973a). this procedure vields very salient dominance differences in virtually all pairings.

\section{RESULTS}

Table 1 shows the win-loss record of each monkey during each of the three dominance tests. Each monkey was ranked on the basis of the total number of pairings in which he successfully dominated his pairmate. This procedure yielded the dominance hierarchies presented in the left-hand column of Table 1. Ties in total wins were resolved by labeling dominant that monkey within the tie which actually dominated the other animals within the tie during those particular pairings.

Examination of the win-loss pattern within each of the three dominance tests indicated that the dominance ability of individual monkeys was highly stable from day to day within each test. Unstable dominance within individual Ss would appear in Table 1 in the form of nonlinear pairings. However, the proportion of nonlinear pairings to total pairings was quite small: $3 / 45,1 / 45$, and $1 / 45$, respectively, in Dominance Tests I, II, and III. The null hypothesis that half the pairings would result in linear outcomes by chance was easily rejected in each case (all binomial $\mathrm{p}<.00001$ ). Thus, one result of the present study was the replication of the cross-sectionally stable linear dominance hierarchy reported for squirrel monkeys by Clark \& Dillon (1973a).

Comparison of the dominance orders from Dominance Test I to Dominance Test II and from Dominance Test II to Dominance Test III revealed a 
pattern of orderly changes of dominance rank within a fairly stable overall dominance structure. For example. rank order correlations between the dominance hierarchies determined at 6-month intervals were quite high in value. The Spearman rank correlation between Dominance Tests I and II was $r_{s}=.92 .(p<.01)$. between Dominance Tests II and III was $r_{s}=.95$. $(p<.01)$, and between I and III was $r_{s}=.85 .(p<.01)$. Consequently, longitudinal dominance relationships between squirrel monkeys may be characterized as relatively stable over long periods of time in the absence of interpolated social contact.

The status changes that were observed in the present study appeared to be both slight and orderly. As can be seen in Table 1,7 of the 10 monkeys in the group experienced a change of one or less ranks in dominance across the three measurement occasions (Ss 154, 165, $158,170,156,171$, and 163). The remaining 3 animals changed status positions in an orderly appearing fashion. Both the intially most dominant male (S 168) and the initially most dominant female (S 162) consistently lost rank from Test I to Test II and from Test II to Test III, with male S 168 occupying the fourth position and female S 162 occupying the seventh position by the completion of the study. Finally, while the initially dominant male (S 168) lost status, the initially fourth dominant male (S 137) consistently gained rank over the repeated tests to occupy the first position by the end of the year. Whetever the explanation for these status changes, it obviously cannot involve the experiences of winning or losing in competitive social situations. The monkeys in the present study were continuously segregated from one another between the dominance tests.

\section{DISCUSSION}

The present experiment was conducted to determine the degree of long-term stability in dominance relationships between squirrel monkeys who had received no interpolated social experiences with other group members. The changes in status positions that were observed during the 12 -month period of time, interrupted by only a single brief dominance measurement, were found to be slight. Thus. in general terms, the dominance hierarchy was found to be highly stable. This result is consistent with previous studies. in which similar stable dominance relations were observed over long spans in pairwise-tested rhesus monkeys (Warren \& Maroney. 1958), and in pigtail macaques, crabeating maceques. gelada baboons, Celebes black apes, and sooty mangabeys (al! Old World primates) living in established groups (Bernstein, 1970). However, the present experiment is the first to demonstrate long-term stable dominance in a New World primate species. the squirrel monkey.

The status position changes involving male monkeys, which were observed in the present study, were very similar to those reported by Baldwin (1968) for a group of free-living squirrel monkeys. As previously mentioned, Baldwin measured several different status hierarchies among the four adult males of his group during a 4-month span of time. Most of these different orders resulted from the rank changes of two of the males, who frequently exchanged occupancy of the first and fourth ranks in the dominance structure of the male group. The primary status changes in the present instance also involved two males, who initially ranked first and fourth but, by the end of the year, had exchanged status positions. An important difference between these two results is that the reversal in ranks observed by Baldwin occurred during a much shorter span of time than was employed in the present study, several weeks as opposed to 12 months. On the other hand, the Baldwin monkeys received continuous social contact throughout his study, while the animals in the present experiment were socially restricted. The obvious conclusion is that dominance status may change "spontaneously" in a very gradual manner, but the addition of social experiences may serve to facilitate or accelerate these changes.

\section{REFERENCES}

Baldwin, J. D. The social behavior of adult male squirrel monkeys (Saimiri sciureus) in a seminatural environment. Folia Primatologica, 1968, 9, 281-314.

Bernstein, I. S. Primate status hierarchies. In L. A. Rosenblum (Ed.), Primate behavior: Developments in field and laboratory research. New York: Academic Press, 1970.

Candland, D. K., Bryan, D. C., Nazar, B. L., Kopf, K. J., \& Sendor, M. Squirrel monkey heart rate during formation of status orders. Journal of Comparative \& Physiological Psychology, 1970, 70, 417-423.

Clark, D. L., \& Dillon, J. E. Evaluation of the water incentive method of social dominance measurement in primates. Folia Primatologica, 1973a, in press.

Clark, D. L., \& Dillon, J. E. Social dominance relationships between previously unacquainted male and female squirrel monkeys. Behaviour, $1973 \mathrm{~b}$, in press.

Ploog, D. W., Blitz, J., \& Ploog, F. Studies on social and sexual behavior of the squirrel monkey (Saimiri sciureus). Folia Primatologica, 1963, 1, 29-66.

Plotnik, R., King, F. A., \& Roberts, L. Effects of competition of the agressive behavior of squirrel and cebus monkeys. Behaviour, 1968, 32, 315-332.

Warrer, J. M., \& Maroney, R. J. Competitive social interaction between monkeys. Journal of Social Psychology, 1958, 48, 223-233.

(Received for publication June 1, 1973.)

\section{Licking response astributions asociated with the acrusition of scheduie-induced polydipsia \\ ROBERT W. SCHAEFFER \\ Auburn University. Auburn. Ala. 36830 \\ and

\author{
University of Kansas. Lawrence. Kans. 66044
}

*Supported in part by Grants MH-08775 and MH-12025 from the National Institute of Mental Health, Robert W. Schaeffer, principal investigator.
The relation between drinking and pellet delivery was examined in a rat that acquired polydipsia when it was exposed to a free fixed-interval (FFI) 50 -sec food (Noyes 45-mg pellets) schedule. A distributional analysis 